\title{
The antimicrobial quantitative relationship and mechanism of plant flavonoids to gram-positive bacteria
}

Ganjun Yuan ( $\sim$ gyuan@jxau.edu.cn )

Jiangxi Agricultural University

Xuexue Xia

Jiangxi Agricultural University

Yingying Guan

Jiangxi Agricultural University

Houqin $\mathrm{Yi}$

Jiangxi Agricultural University

Shan Lai

Jiangxi Agricultural University

Yifei Sun

Jiangxi Agricultural University

Seng Cao

Jiangxi Agricultural University

\section{Research Article}

Keywords:

Posted Date: January 31st, 2022

DOI: https://doi.org/10.21203/rs.3.rs-1313674/v1

License: (c) (i) This work is licensed under a Creative Commons Attribution 4.0 International License.

Read Full License 


\section{Abstract}

Antimicrobial resistance (AMR) poses a serious threat to human health, and new antimicrobial agents are desperate in need. Plant flavonoids have been increasingly paid attention to, for their antibacterial activities, combinational effects on enhancing the antibacterial activity, and reversing the AMR. Based on our previous work, another two regression equations were established once again between the minimum inhibitory concentrations (MICs) (y) and the lipophilicity parameters ACD/LogP or LogD (x). After comparing the fitting goodness of four equations including two published by us, the best one, regarded as the antimicrobial quantitative relationship of plant flavonoids to gram-positive bacteria, was $y=$ $-0.1285 \times 6+0.7944 \times 5+51.785 \times 4-947.64 \times 3+6638.7 \times 2-21273 x+26087$, and here $x$ is the LogP value. From this equation, the MICs of most plant flavonoids to gram-positive bacteria can be calculated. The minimum MIC was also predicted as approximately $0.9644 \mu \mathrm{M}$, and likely from 0.24 to $0.96 \mu \mathrm{M}$. Further, this more reliable equation further proved that the lipophilicity is a key factor of plant flavonoids against gram-positive bacteria. Combined with the antibacterial mechanism suggested in our previous work and the support from recent literature, more intuitive evidence powerfully confirmed the antibacterial mechanism that the cell membrane is the major site of plant flavonoids acting on gram-positive bacteria, and that involving the damage of phospholipid bilayers.

\section{Introduction}

Antimicrobial resistance (AMR) has been becoming a serious threat to the public health, and the COVID19 pandemic would further accelerate this global problem ${ }^{1}$. So, new antimicrobial agents are in desperate need $^{2-3}$. When antibiotics have been used for the treatment on bacterial infection, most of them will also bring about some adverse reactions, and eventually be resistance in clinic ${ }^{4}$. However, some plant metabolites having moderate antimicrobial activities, being nontoxic to human body, can enhance the antibacterial activity of some antibiotics, and even reverse the AMR ${ }^{5}$. Among them, plant flavonoids have been paid close attention to. Some structure-activity relationships of them against bacteria were sporadically summarized ${ }^{5-7}$. However, a universality conclusion remains unclear.

In our previous work ${ }^{8}$, two regression equations were established for predicting the antibacterial activities of plant flavonoids to gram-positive bacteria, based on the data pairs of the physicochemical parameter $\mathrm{ACD} / \log \mathrm{P}$ or $\log _{7.40}$ and the minimum inhibitory concentrations (MICs) of sixty-six flavonoids reported $^{9-14}$. Subsequently, these two equations were further verified using those of another sixty-eight flavonoids reported ${ }^{5,15-20}$. Combined literature analysis, we concluded that the lipophilicity is a key factor for flavonoids against gram-positive bacteria, and the cell membrane is the major action site ${ }^{8}$.

Here these data were reanalyzed, and two regression equations were reestablished based on the physicochemical parameter $\mathrm{ACD} / \log \mathrm{P}$ or $\mathrm{Log}_{7.40}$ and the average MICs (or $\mathrm{MIC}_{90}$ ) to various grampositive bacteria, of all flavonoids in our previous work ${ }^{8}$. According to statistical procedure and comparison with those two equations previously reported, a regression equation with a lager correlation 
coefficient $(r)$ of 0.9703 was eventually proved as the best one for fitting the correlation between the antibacterial activity and the lipophilicity. This equation shows more accurate and more reliable, and can be practically considered as the quantitative relationship of plant flavonoids against gram-positive bacteria. Moreover, the regression curves established from the $\log _{10}(\mathrm{MIC})$ and the $\log P\left(\operatorname{or} \log _{7.40}\right.$ ) provides a more intuitive fact for the direct correlation between the antibacterial activity to gram-positive bacteria and the lipophilicity, and for the antibacterial mechanism of plant flavonoids acting on the cell membrane. These above were diagrammatically presented as Fig. 1.

\section{Results}

Structure, antibacterial activity, and physicochemical parameter. One hundred and thirty-four flavonoids reported in twelve papers were reorganized by us ${ }^{8,9-20}$, ninety-two compounds were screened out, according to the procedure in section Method, for subsequent regression analyses. These flavonoids involve twelve subclasses, and which mainly include flavones, dihydroflavones, flavonols, dihydroflavonols, isoflavones, dihydroisoflavones, dihydroisoflavane and chalcones. Their chemical structures were shown on Fig. 1 to 6 , and 8 in our previous work ${ }^{8}$, and the numbers of these compounds remains unchanged. Their antimicrobial activities (MICs) and physicochemical parameters ( $\mathrm{ACD} / \log \mathrm{P}$ and $\log _{7.40}$ ) were reorganized and listed in Table 1. If possible, the average MIC or $\mathrm{MIC}_{90}$ of a certain flavonoid to different pathogenic bacteria was considered as its MIC. In other cases, the MIC of a certain flavonoid to pathogenic bacteria was processed according to the rules in section Method.

Regression equation from the MICs and the physicochemical parameters. The regression analyses for the MICs $(y)$ to gram-positive bacteria and the physicochemical parameters $\operatorname{LogP}$ or $\log _{7.40}(x)$, of these flavonoids were achieved. Two regression curves were shown on Fig. 1, and two regression equations together with their correlation coefficients $(r)$ were calculated and shown on Fig. 1 and in Table 2.

From Fig. 1, the characteristics of these two regression curves were similar to those established from sixty-six flavonoids ${ }^{8}$. However, they presented larger correlation coefficient ( $r$ ) values (Table 2), and which better indicated that the significant correlation between the physicochemical parameter LogP or $\log _{7.40}$ and the MICs of plant flavonoids to gram-positive bacteria, especially for that between the LogP and the MICs as which presented a lager correlation coefficient ( $r$ ) value of 0.9703 (Table 2). Thereby, these two equations have a greater potency to further prove that the antibacterial activities of plant flavonoids to gram-positive bacteria is close related to their lipophilicities.

Antimicrobial quantitative relationship. Including two regression equations reported by us $^{8}$, four regression equations were established for the fitting of the correlation between the antimicrobial activity (MIC) and the physicochemical parameter ( $\log P$ or $\left.\log D_{7.40}\right)$. To compare the fitting goodness of these four equations, two statistical parameters, coefficient of determination $\left(R^{2}\right)$ and residual standard deviation (s), were respectively calculated, and presented in Table 3. Generally, the closer the $\boldsymbol{R}^{2}$ is to 1 , 
the higher the goodness of fit, and the closer the predictive value is, on the whole, to the actual one. The smaller $\boldsymbol{s}$ is, the smaller the mean deviation between the predictive value and the actual one.

From Table 3, the largest value of the $\boldsymbol{R}^{2}(0.9413)$ and the smallest value of $\boldsymbol{s}(68.1127)$ indicated that equation (1) is more reliable and is the best one for fitting the quantitative relationship between the physicochemical parameter and the antimicrobial activity to gram-positive bacteria, of plant flavonoids. Thereout, the MICs of most plant flavonoids to gram-positive bacteria can be roughly calculated from this equation by substituting their LogP values (calculated by ACD/Labs 6.0) into.

Table 1. Plant flavonoids together with their structure types, physicochemical parameters and antimicrobial activities, used for the regression analyses ${ }^{8}$. 


\begin{tabular}{|c|c|c|c|c|c|}
\hline Compounds ${ }^{a}$ & Structure types & $\log P^{b}$ & $\log _{7.40^{b}}^{b}$ & $\operatorname{MIC}(\mu M)^{c}$ & $\log _{10}(M I C)^{c}$ \\
\hline 2 & & 5.09 & 4.92 & 11.3 & 1.0531 \\
\hline 3 & & 7.02 & 6.8 & 8.85 & 0.9469 \\
\hline 4 & & 5.29 & 5.09 & 14.7 & 1.1673 \\
\hline 6 & & 7.02 & 6.81 & 23.7 & 1.3747 \\
\hline 7 & & 4.18 & 4.09 & 25.9 & 1.4133 \\
\hline 8 & & 4.18 & 3.98 & 25.9 & 1.4133 \\
\hline 9 & & 5.74 & 5.5 & 22.7 & 1.3560 \\
\hline 10 & & 6.52 & 6.33 & 5.9 & 0.7709 \\
\hline 11 & & 6.30 & 6.08 & 5.7 & 0.7559 \\
\hline 12 & & 7.05 & 6.83 & 5.5 & 0.7404 \\
\hline 13 & & 7.27 & 7.09 & 5.7 & 0.7559 \\
\hline 16 & & 7.24 & 7.06 & 9.15 & 0.9614 \\
\hline 17 & & 4.56 & 4.37 & 10.5 & 1.0212 \\
\hline 20 & & 5.56 & 5.34 & 52.8 & 1.7226 \\
\hline 21 & & 6.54 & 6.32 & 9.15 & 0.9614 \\
\hline 22 & & 6.61 & 6.39 & 11.35 & 1.0550 \\
\hline 23 & & 5.18 & 4.96 & 85.05 & 1.9297 \\
\hline 24 & & 6.25 & 5.97 & 8.05 & 0.9058 \\
\hline 25 & & 7.02 & 6.81 & 13.65 & 1.1351 \\
\hline 26 & & 7.32 & 7.12 & 10.6 & 1.0253 \\
\hline 27 & & 6.72 & 6.51 & 20.4 & 1.3096 \\
\hline 28 & & 3.27 & 3.04 & 233.7 & 2.3687 \\
\hline 29 & & 4.60 & 4.38 & 84.4 & 1.9263 \\
\hline 30 & & 4.27 & 4.05 & 84.1 & 1.9248 \\
\hline 31 & & 4.67 & 4.46 & 186.4 & 2.2704 \\
\hline 32 & & 6.10 & 5.76 & 107.3 & 2.0306 \\
\hline 33 & & 5.63 & 5.29 & 113.6 & 2.0554 \\
\hline 34 & & 4.52 & 3.84 & 140.2 & 2.1467 \\
\hline
\end{tabular}




\begin{tabular}{|c|c|c|c|c|}
\hline 35 & 4.52 & 3.93 & 140.2 & 2.1467 \\
\hline 36 & 6.20 & 5.53 & 73 & 1.8633 \\
\hline 37 & 6.72 & 6.51 & 9.5 & 0.9777 \\
\hline 38 & 7.32 & 7.12 & 14.75 & 1.1688 \\
\hline 39 & 8.75 & 8.54 & 24.45 & 1.3883 \\
\hline 40 & 7.32 & 7.13 & 24.6 & 1.3909 \\
\hline 41 & 5.94 & 5.75 & 90.8 & 1.9581 \\
\hline 42 & 7.97 & 7.78 & 19 & 1.2788 \\
\hline 43 & 6.74 & 6.5 & 37.9 & 1.5786 \\
\hline 44 & 8.84 & 8.64 & 12.25 & 1.0881 \\
\hline 45 & 3.79 & 3.67 & 251.75 & 2.4010 \\
\hline 46 & 3.79 & 3.53 & 167.8 & 2.2248 \\
\hline 47 & 3.92 & 3.59 & 42.1 & 1.6243 \\
\hline 48 & 4.67 & 4.35 & 61 & 1.7853 \\
\hline 49 & 4.11 & 3.67 & 84.5 & 1.9269 \\
\hline 52 & 4.51 & 4.27 & 87.8 & 1.9435 \\
\hline 53 & 2.42 & 2.11 & 1734.6 & 3.2392 \\
\hline 54 & 4.64 & 4.34 & 88.3 & 1.9460 \\
\hline 55 & 6.52 & 6.33 & 11.05 & 1.0434 \\
\hline 56 & 8.76 & 8.7 & 9 & 0.9542 \\
\hline 57 & 4.72 & 4.51 & 24.25 & 1.3847 \\
\hline 58 & 6.52 & 6.33 & 14.7 & 1.1673 \\
\hline 59 & 5.89 & 5.67 & 17.75 & 1.2492 \\
\hline 60 & 5.89 & 5.68 & 21.3 & 1.3284 \\
\hline 61 & 6.60 & 6.35 & 22.05 & 1.3434 \\
\hline 62 & 5.81 & 5.62 & 28.4 & 1.4533 \\
\hline 63 & 5.81 & 5.62 & 28.4 & 1.4533 \\
\hline 64 & 4.56 & 4.37 & 35.1 & 1.5453 \\
\hline 66 & 3.19 & 2.96 & 734.6 & 2.8661 \\
\hline
\end{tabular}




\begin{tabular}{|lllll|}
67 & 4.20 & 3.77 & 184.7 & 2.2665 \\
\hline Continued & & & & \\
\hline
\end{tabular}




\begin{tabular}{|c|c|c|c|c|c|}
\hline Compounds ${ }^{a}$ & Structure types & $\log P^{b}$ & $\log _{7.40}^{b}$ & $\operatorname{MIC}(\mu M)^{c}$ & $\log _{10}(M I C)^{c}$ \\
\hline 70 & & 3.1 & 2.32 & 670.5 & 0.7597 \\
\hline 72 & & 7.33 & 6.89 & 5.75 & 2.9485 \\
\hline 73 & & 2.83 & 2.16 & 888.1 & 1.5653 \\
\hline 75 & & 8.63 & 8.17 & 36.75 & 0.7284 \\
\hline 76 & & 6.59 & 6.4 & 5.35 & 0.8325 \\
\hline 77 & & 6.6 & 6.42 & 6.8 & 1.4518 \\
\hline 81 & & 4.95 & 4.82 & 28.3 & 1.1508 \\
\hline 82 & & 4.95 & 4.82 & 14.15 & 1.5502 \\
\hline 86 & & 5.67 & 5.07 & 35.5 & 2.5715 \\
\hline 87 & & 3.15 & 2.91 & 372.8 & 2.1166 \\
\hline 88 & & 5.38 & 5.12 & 130.8 & 1.7239 \\
\hline 89 & & 4.15 & 3.48 & 52.95 & 1.0434 \\
\hline 91 & & 6.32 & 6.32 & 11.05 & 1.4031 \\
\hline 92 & & 4.41 & 4.4 & 25.3 & 1.4609 \\
\hline 93 & & 4.18 & 4.18 & 28.9 & 1.7649 \\
\hline 94 & & 6.64 & 6.63 & 58.2 & 3.2186 \\
\hline 97 & & 2.62 & 1.95 & 1654.3 & 2.5505 \\
\hline 113 & & 3.23 & 3.1 & 355.2 & 2.6985 \\
\hline 114 & & 3.4 & 3.26 & 499.5 & 2.1318 \\
\hline 115 & & 5.03 & 4.48 & 135.45 & 2.1649 \\
\hline 116 & & 4.63 & 4.07 & 146.2 & 2.2399 \\
\hline 118 & & 5.69 & 5.4 & 45.41 & 1.2940 \\
\hline 119 & & 7.33 & 7.16 & 19.68 & 1.8510 \\
\hline 120 & & 5.24 & 4.69 & 70.95 & 2.2398 \\
\hline 121 & & 4.7 & 4.27 & 173.7 & 1.5786 \\
\hline 122 & & 7.13 & 6.89 & 37.9 & 2.1149 \\
\hline 123 & & 4.56 & 4.27 & 130.3 & 2.1318 \\
\hline 124 & & 5.47 & 5.21 & 135.45 & 1.9557 \\
\hline
\end{tabular}




\begin{tabular}{|lllll|}
125 & 5.47 & 5.21 & 90.3 & 2.0964 \\
126 & 4.83 & 4.67 & 124.85 & 1.2765 \\
127 & 6.69 & 6.5 & 18.9 & 1.6375 \\
128 & 5.99 & 5.98 & 43.4 & 2.5224 \\
130 & 5.61 & 5.59 & 65.15 & 1.6721 \\
133 & 4.1 & 4.1 & & 47 \\
\hline
\end{tabular}

a: The chemical structures of flavonoids showed in our previous work ${ }^{8}$.

b. The $\log P$ and $\log D_{7.40}$ values were calculated using software ACD/Labs 6.0.

c: MIC, minimum inhibitory concentration, and here a processed MIC of a certain flavonoid to various gram-positive including Staphylococcus aureus, S. epidermidis, or/and Bacillus subtilis, etc. was presented; $\log _{10}(\mathrm{MIC})$ means $\log _{10}$ of MIC.

Table 2. Regression equations for the correlation between the physicochemical parameter $(x)$ and the antimicrobial activity $(y)$ to gram-positive bacteria, of plant flavonoids ${ }^{a}$

\begin{tabular}{|c|c|c|c|}
\hline $\begin{array}{l}\text { Equation } \\
\text { number }\end{array}$ & $\begin{array}{l}\text { Sample } \\
\text { numbers } \\
\text { (n) }\end{array}$ & $\begin{array}{l}\text { Parameters } \\
\text { (x) }\end{array}$ & Regression equation $\left(\boldsymbol{c}^{c}\right)$ \\
\hline (1) & 92 & $\log P$ & $\begin{array}{l}y=-0.1285 x^{6}+0.7944 x^{5}+51.785 x^{4}-947.64 x^{3}+ \\
6638.7 x^{2}-21273 x+26087(0.9703)\end{array}$ \\
\hline (2) & 92 & $\log _{7.40}$ & $\begin{array}{l}y=0.2337 x^{6}-9.1209 x^{5}+146.54 x^{4}-1240.3 x^{3}+ \\
5837.4 x^{2}-14534 x+15094(0.9462)\end{array}$ \\
\hline (3) & $66^{d}$ & $\log P$ & $\begin{array}{l}y=-1.6745 x^{5}+56.143 x^{4}-741.93 x^{3}+4831.8 x^{2}-15531 x \\
+19805(0.9349)\end{array}$ \\
\hline (4) & $66^{d}$ & $\log _{7.40}$ & $\begin{array}{l}y=-1.1474 x^{5}+38.802 x^{4}-515.39 x^{3}+3361.9 x^{2}-10789 x \\
+13706(0.9309)\end{array}$ \\
\hline
\end{tabular}

a. The antimicrobial activity $(y)$ was the mean MIC (or $\mathrm{MIC}_{90}$ ) of a certain flavonoid to gram-positive bacteria mainly including Staphylococcus aureus, S. epidermidis and Bacillus subtilis.

b. The physicochemical parameter $(x)$ was calculated using software ACD/Labs 6.0. 
c. $r$, correlation coefficients; the significant level $a$ was set as 0.01 , and the critical value of $r_{0.995}(90)$ and $r_{0.995}(64)$ were equal to 0.27 and 0.32 , respectively.

d: The regression equations were established in our previous work $^{8}$.

Table 3. Fitting goodness of the regression equations ${ }^{\mathbf{a}}$

\begin{tabular}{|llll|}
\hline Equation number & $\begin{array}{l}\text { Coefficient of determination } \\
\left(\boldsymbol{R}^{2}\right)\end{array}$ & $\begin{array}{l}\text { Residual standard deviation } \\
(\boldsymbol{s})\end{array}$ & Goodness of fit \\
\hline$(1)$ & 0.9413 & 68.1127 & The best \\
\hline$(2)$ & 0.8949 & 91.1187 & - \\
\hline$(3)$ & 0.8740 & 89.5452 & - \\
\hline$(4)$ & 0.8666 & 92.1391 & - \\
\hline
\end{tabular}

a. Equations (3) and (4) were reported in our previous work ${ }^{8}$, and thereout the $\boldsymbol{R}^{2}$ and $\boldsymbol{s}$ values were calculated from the sixty-six data pairs.

Regression equation from the $\log _{10}(\mathrm{MIC})$ and the physicochemical parameter. Base on the regression equations previously established and literature analyses, the antibacterial mechanism of plant flavonoids acting on the cell membrane of gram-positive bacteria was suggested by $u^{8}{ }^{8}$. To better observe the correlation between the antibacterial activity and the lipophilic parameters, the regression analyses for the common logarithm $\left(\log _{10}\right)$ of MIC $(y)$ to gram-positive bacteria and the LogP or $\log _{7.40}(x)$, of these plant flavonoids, was further performed. Their regression curves and regression equations, with the correlation coefficient $(r)$ values of 0.8040 and 0.8212 , were respectively shown on Fig. 2.

Both $r$ values are greater than 0.27 which was the critical value when $a$ was set at 0.01 and the sample number was 92 . This indicates that there is a very significant correlation between the $\log _{10}(\mathrm{MIC})$ and the $\operatorname{LogP}$ or $\log _{7.40}$. Along with the increase of the $\log P$ or $\log _{7.40}$ value in an approximate range from 2.0 to 8.0 , the $\log _{10}(\mathrm{MIC})$ value will decrease, namely the antibacterial activities will increase. These, more intuitively, demonstrated that the antibacterial activity of plant flavonoids to gram-positive bacteria is directly related to their lipophilicity.

\section{Discussion And Conclusion}


Flavonoids are a class of secondary metabolites widely distributing in various parts of the plant, and so far, approximate 10,000 compounds have been discovered. These plant flavonoids have various bioactivities, such as antioxidation, antibiosis, estrogen-like effect, and prevention and treatment of cardiovascular diseases ${ }^{5,7,21}$. After some of them were discovered to have a potency enhancing the antibacterial effect of some antibiotics, or reversing the $\mathrm{AMR}^{5,6}$, their antibacterial activities have been increasingly paid close attention to.

Based on our previous report ${ }^{8}$, here the correlation between the $A C D / \operatorname{LogP}$ or $\log _{7.40}$ values and the MICs to gram-positive bacteria, of plant flavonoids were further proved by a lager sample $(n=92)$, and both $r$ values ( 0.9703 and 0.9462 ) of two regression equations were lager than those previously published. Thereby, these were better and more scientific to prove that there is a direct correlation between the lipophilicity and the antibacterial activity, of plant flavonoids. The statistical evaluation procedure, including the calculation and comparison of $\boldsymbol{R}^{2}$ and $\boldsymbol{s}$ (Table 3 ), concluded that equation (1) as $y=-0.1285 x^{6}+0.7944 x^{5}+51.785 x^{4}-947.64 x^{3}+6638.7 x^{2}-21273 x+26087$, between the LogP value $(x)$ and the MICs $(y)$, is the most reliable and the best one. More importantly, this equation was established on the data of twelve subclasses of flavonoids including seven main subclasses, while those previously published were generated from the data of three subclasses of flavonoids. Thereby, these above together indicated that equation (1) is more scientific, reliable and universal, and can be considered as the antimicrobial quantitative relationship of plant flavonoids to gram-positive bacteria.

As previously reported by Cushnie ${ }^{6}$, many factors, involving the MIC of determination methods and details, may influence on the experimental MIC value, and the antibacterial activities of a compound to different pathogens are varied ${ }^{8}$. Moreover, the LogP value calculated by soft ACD/Labs 6.0 generally was presented as a range. Therefore, the tested MICs would fluctuate within a reasonable range of the actual values, and so the determined MICs would probably range from $1 / 2 x$ to $2 x$ the predicted one, more likely from $1 / 4 \times$ to $4 \times$ the predicted one, since the MICs were generally tested by double dilution method ${ }^{22}$. Based on these, the MIC (More accurately, $\mathrm{MIC}_{90}$ ) for a certain compound of flavonoids to gram-positive bacteria can be roughly predicted through the calculation by substituting its ACD/LogP value into equation (1). Furthermore, the minimum MIC of plant flavonoids to gram-positive bacteria can be predicted as approximately $0.9644 \mu \mathrm{M}$, and at the time the LogP value of is equal to about 7.055. Considering that the experimental MICs would fluctuate, the minimum MIC tested of plant flavonoids to gram-positive bacteria would more likely range from 0.24 to $0.96 \mu \mathrm{M}$.

The MICs of most plant flavonoids to gram-positive bacteria can be correctly predicted from this equation, and even if those flavonoid subclasses were not included when equation (1) was established. For example, the MIC of $a$-mangostin, a xanthone from mangosteen, against gram-positive bacteria was predicted as $8.16 \mu \mathrm{M}(3.35 \mu \mathrm{g} / \mathrm{mL})$, and so the MICs tested were deduced to range from 0.84 to 13.4 $\mu \mathrm{g} / \mathrm{mL}$. This is, by and large, in consistent with the determined MIC value of 1 or $0.5 \mu \mathrm{g} / \mathrm{mL}(0.5 \mu \mathrm{g} / \mathrm{mL}$ for $\left.\mathrm{MIC}_{90}\right)^{23}$, and also approved by the antibacterial tests repeated by two students at different time to $S$. aureus ATCC 25923 in our Laboratory $(0.5,1$ or $2 \mu \mathrm{g} / \mathrm{mL}$ for the MICs). Of course, a few of plant 
flavonoids probably present incorrect predictions, such as baicalein, a rare 5,6,7-trihydroxyl flavonoid from Scutellaria baicalensis. However, the structural modification of baicalein for increasing the lipophilicity of molecules will increase the antibacterial activity ${ }^{24}$. This indicated that the correlation between the antibacterial activity and the lipophilicity is also suitable for baicalein.

Considering that equation (1) was established from the data pairs of the LogP values in an approximate range of 2.4 to 8.9 , and that there are few data pairs in the range of 8.0 to 8.9 when equation (1) was established, the antimicrobial quantitative relationship of plant flavonoids to gram-positive bacteria is more scientifical and reliable when used for the antibacterial prediction of plant flavonoids having the LogP value ranged from 2.4 to 8.5. However, this does not exclude that the antibacterial activity of some flavonoids, having the LogP value outside of this range, can be accurately predicted using this equation. Empirically, the LogP can be extended to the range from 2.0 to 8.5 .

Moreover, the regression equation established by the parameter LogP showed better fit for the correlation between the antibacterial activity and the lipophilicity than that by the $\log _{7.40}$ (Table 3). However, the $\log _{7.40}$ can be regarded as the LogP at pH 7.40, and is better to reflect the actual state of a compound in the medium of MIC determination ${ }^{22}$. So, the MICs calculated from equation (2), are also references for the antibacterial prediction of plant flavonoids although this equation established by the parameter $\log _{7.40}$ is not the best one for the antibacterial prediction. Similar to our previous analyses ${ }^{8}$, the more reliable regression equations for a certain subclass of flavonoids, with a larger correlation coefficient $(r)$, can be also established for the more accurate prediction of the MICs according to similar procedure, and for the structural design and optimization to obtain more efficient antibacterial activity. It is worth noting that this equation is not necessarily suitable for the antibacterial prediction of all structural derivatives of plant flavonoids, especially those introducing heteroatoms, such as nitrogen and halogen.

As we suggested ${ }^{8}$, the cell-membrane is the major site of plant flavonoids acting on gram-positive bacteria, and which likely involving the disruption or damage of phospholipid bilayers. This was further supported by recent reports ${ }^{23,25,26}$. Here, more reliable regression equations (1) and (2) further proved that the lipophilicity is a key factor responsible for the antibacterial activity of plant flavonoids to grampositive bacteria. Powerfully, these together with the regression analyses for the correlation between the $\log _{10}(\mathrm{MIC})$ and the $\log P$ or $\log _{7.40}$ more intuitively confirmed this antibacterial mechanism of plant flavonoids acting on the cell membrane of gram-positive bacteria.

In conclusion, the MICs $(y)$ of most plant flavonoids to gram-positive bacteria can be calculated by substituting their physicochemical parameter ACD/LogP $(x)$ into the equation $y=-0.1285 x^{6}+0.7944 x^{5}+$ $51.785 x^{4}-947.64 x^{3}+6638.7 x^{2}-21273 x+26087$. Combined with the antibacterial mechanism suggested by us and the support from recent literature, more reliable equations further proved that the lipophilicity is a key factor of plant flavonoids against gram-positive bacteria, and more intuitive evidence powerfully confirmed the antibacterial mechanism that the cell membrane is the major site of plant flavonoids acting on gram-positive bacteria, and which likely involves the damage of phospholipid 
bilayers. Based on these, the antibacterial activity and mechanism of plant flavonoids against grampositive bacteria were diagrammatically presented as Fig. 1, and some errors in Fig. 9 of our published paper happened to be corrected. Simultaneously, all these above provide good references for exploring the antibacterial activity and mechanism of plant flavonoids against gram-negative bacteria.

\section{Methods}

Data and processing. Based on all the data of plant flavonoids in our previous work ${ }^{8}$, the data processing was performed. As no clear MIC value was presented for many flavonoids used for the verification of two regression equations in that paper, such as the MIC of compound $\mathbf{8 4}$ expressed as more than one hundred $(\varangle 100 \mu \mathrm{M})$, these data were processed according to the rules as follows: 1$)$ Discard all ambiguous data which the MICs expressed as more than a certain value; 2) For the MICs expressed as more than or equal to a certain value, the boundary value is considered as the MIC, such as the MIC for compound 69 as $636.4 \mu \mathrm{M}$; 3) For the MICs expressed as more than a range, the latter is considered as the MIC because it is a clear MIC value, such as the MIC for compound 73 as $888.1 \mu \mathrm{M}$; 4) For the MIC expressed as a range, the mean of two boundary values is considered as the MIC because these two boundary values are the MICs of a certain flavonoid to different pathogenic strains, such as the MIC for compound 71 as $520.3 \mu \mathrm{M}$. Finally, based on the variation tendency of MIC along with lipophilicity parameter LogP or LogD, reported by us, and in view of probable fluctuation at the determination of MICs, the probably outliers were discarded using scatter diagram. All the rest data were used as the data of this article for the subsequent analyses. The physicochemical parameter $\log P$ or $\log D_{7.40}\left(\log _{10}\right.$ of oil/water distribution coefficient at $\mathrm{pH}$ 7.40) was calculated using software ACD/Labs 6.0.

Regression analyses. For establishing the antimicrobial quantitative relationship of plant flavonoids to gram-positive bacteria, the regression analyses between the MICs $(y)$ and the physicochemical parameter $\operatorname{LogP}$ or $\log _{7.40}(x)$ were performed using Microsoft Excel software, and the $r$ value was also calculated. To discover more powerful evidence for supporting the antibacterial mechanism of plant flavonoids acting on the cell membrane of gram-positive bacteria, the MIC was further transformed to the $\log _{10}(\mathrm{MIC})$, and subsequently the regression analyses between the $\log _{10}(\mathrm{MIC})(y)$ and the physicochemical parameter $\operatorname{LogP}$ or $\log _{7.40}(x)$ were further achieved.

Statistical analysis. To ensure which one is more reliable, further statistical analyses were performed for all the regression equations including those reported in our previous paper ${ }^{8}$. In the process, two statistical parameters, coefficient of determination $\left(\boldsymbol{R}^{2}\right)$ and residual standard deviation $(\boldsymbol{s})$, were respectively calculated according to equations (5) and (6).

$$
\begin{array}{ll}
R^{2}=1-\frac{\sum\left(y_{i}-\hat{y}_{i}\right)^{2}}{\sum\left(y_{i}-\bar{y}\right)^{2}} & \text { equation (5) } \\
s=\sqrt{\frac{\sum\left(y_{i}-\hat{y}_{l}\right)^{2}}{n-2}} & \text { equation (6) }
\end{array}
$$


Where is the MIC of a certain flavonoid $\boldsymbol{i}$, Correspondingly, $\widehat{y}_{\imath}$ is the prediction MIC of flavonoid $\boldsymbol{i}^{\bar{y}}$ is the mean MIC of all flavonoids in Table 1 , and $n(n=92)$ is the number of all flavonoids.

When comparing the goodness of fit of these regression curves, the closer the $R^{2}$ is to 1 , the higher the goodness of fit, and the closer the predictive value is to the actual one, on the whole. The smaller $\boldsymbol{s}$ is, the smaller the mean deviation between the predictive value and the actual one. Generally, a consistent result will be presented from these two statistical parameters.

\section{Data Availability}

The datasets generated during and/or analyzed during the current study are available from the corresponding author on reasonable request.

\section{Declarations}

\section{Acknowledgements}

This work was financially supported by grants from the National Natural Science Foundation of China (No. 82073745 and 81960636) and the Natural Science Foundation of Jiangxi Province, China (Grant No. 20202BABL206156).

\section{Author contributions}

G.Y. initiated the project, hypothesis and design, analyzed the data and obtained regression equations, wrote the manuscript text, took part in the search and collection of flavonoids data, and prepared the figures and tables; X.X. and Y.G. performed the search and collection of flavonoids data, and took part in the preparation of figures and tables; H.Y., S.L., Y.S. and S.C. took part in the data processing and analysis. All authors reviewed the manuscript.

\section{Data Availability}

The datasets generated during and/or analyzed during the current study are available from the corresponding author on reasonable request.

\section{Additional Information}

Competing Interests: The author declare that they have no competing interests. 


\section{References}

1. Murray, C. J. L. et al. Global burden of bacterial antimicrobial resistance in 2019: a systematic analysis. The Lancet, (2022). https://doi.org/10.1016/S0140-6736(21)02724-0

2. Laxminarayan, R., Sridhar, D., Blaser, M. \& Wang, M. \& Woolhouse, M. Achieving global targets for antimicrobial resistance. Science, 353, 874-875 (2016).

3. Kurosu, M., Siricilla, S. \& Mitachi, K. Advances in MRSA drug discovery: where are we and where do we need to be? Exp. Opin. Drug Discov. 8, 1095-1116 (2013).

4. Xu, X., Xu, L., Yuan, G., Wang, Y., Qu, Y. \& Zhou, M. Synergistic combination of two antimicrobial agents closing each other's mutant selection windows to prevent antimicrobial resistance. Sci. Rep. 8, 7237 (2018).

5. Górniak, I., Bartoszewski, R. \& Króliczewski, J. Comprehensive review of antimicrobial activities of plant flavonoids. Phytochem. Rev. 18, 241-272 (2019).

6. Cushnie, T. P. T. \& Lamb, A. J. Antimicrobial activity of flavonoids. Int. J. Antimicrob. Agents 26, 343356; doi:10.1016/j.ijantimicag.2005.12.002 (2005).

7. Xie, Y., Yang, W., Tang, F., Chen, X. \& Ren, L. Antibacterial activities of flavonoids: structure-activity relationship and mechanism. Curr. Med. Chem. 22, 132-149; doi:

$10.2174 / 0929867321666140916113443$ (2015).

8. Xu, X. et al. Synergistic combination of two antimicrobial agents closing each other's mutant selection windows to prevent antimicrobial resistance. Sci. Rep. 8, 7237; doi:10.1038/s41598-018-25714-z (2018).

9. Kuroyanagi, M., Arakawa, T., Hirayama, Y. \& Hayashi T. Antibacterial and antiandrogen flavonoids from Sophora flavescens. J. Nat. Prod. 62, 1595-1599 (1999).

10. Šmejkal, K. et al. Antibacterial C-geranylflavonoids from Paulownia tomentosa fruits. J. Nat. Prod. 71, 706-709 (2008).

11. Navrátilová, A. et al. Minor C-geranylated flavanones from Paulownia tomentosa fruits with MRSA antibacterial activity. Phytochemistry 89, 104-113 (2013).

12. Inui, S. et al. Solophenols B-D and solomonin: new prenylated polyphenols isolated from propolis collected from the solomon islands and their antibacterial activity. J. Agric. Food Chem. 60, 11765-11770 (2012).

13. Sasaki, H., Kashiwada, Y., Shibata, H. \& Takaishi, Y. Prenylated flavonoids from Desmodium caudatum and evaluation of their anti-MRSA activity. Phytochemistry 82, 136-142 (2012). 
14. Tsuchiya, H., Sato, M. et al. Comparative study on the antibacterial activity of phytochemical flavanones against methicillin-resistant Staphylococcus aureus. J. Ethnopharmacol. 50, 27-34 (1996).

15. Edziri, H. et al. Antibacterial, antifungal and cytotoxic activities of two flavonoids from Retama raetam flowers. Molecules 17, 7284-7293; doi:10.3390/molecules17067284 (2012).

16. Sufian, A. S., Ramasamy, K., Ahmat, N., Zakaria, Z.A. \& Yusof, M. I. M. Isolation and identification of antibacterial and cytotoxic compounds from the leaves of Muntingia calabura L. J. Ethnopharmacol. 146, 198-204 (2013).

17. Fukai, T. et al. Antimicrobial activity of licorice flavonoids against methicillin-resistant Staphylococcus aureus. Fitoterapia 73, 536-539 (2002).

18. Fukai, T., Marumo, A., Kaitou, K., Kanda, T., Terada, S. \& Nomura T. Anti-Helicobacter pylori flavonoids from licorice extract. Life Sci. 71, 1449-1463 (2002)

19. Ohemeng, K. A., Schwender, C. F., Fu, K. P. \& Barrett, J. F. DNA gyrase inhibitory and antibacterial activity of some flavones(I). Bioorg. Med. Chem. Lett. 3, 225-230 (1993).

20. Hatano, T. et al. Phenolic constituents of Licorice. VIII. ${ }^{1)}$ Structures of glicophenone and glicoisoflavanone, and effects of Licorice phenolics on methicillin-resistant Staphylococcus aureus. Chem. Pharm. Bull. 48, 1286-1292 (2000). https://doi.org/10.1248/cpb.48.1286

21. Veitch, N. C. \& Grayer, R. J. Flavonoids and their glycosides, including anthocyanins. Nat. Prod. Rep. 21, 539-573 (2004).

22. Clinical and Laboratory Standards Institute. Methods for Dilution Antimicrobial Susceptibility Tests for Bacteria That Grow Aerobically: Approved Standard. 19 ${ }^{\text {th }}$ Edn. CLSI Document M07-A9. Clinical and Laboratory Standards Institute, Wayne, Pennsylvania, USA (2012).

23. Song, M., Liu, Y., Li T., Liu X., Hao Z., Ding S., Panichayupakaranant, P., Zhu K., Shen J. Plant natural flavonoids against multidrug resistant pathogens. Adv. Sci. 8, 2100749 (2021).

24. Wang, S. et al. Synthesis and biological evaluation of novel 7-0-lipophilic substituted baicalein derivatives as potential anticancer agents. Med. Chem. Commun. 6, 1864-1873 (2015).

25. Koh, J. J. et al. et al. Rapid bactericidal action of $a$-mangostin against MRSA as an outcome of membrane targeting. Biochim. Biophys. Acta 1828, 834-844 (2013).

26. Zou, H. et al. Design and Synthesis of Amphiphilic Xanthone-Based, Membrane-Targeting Antimicrobials with Improved Membrane Selectivity. J. Med. Chem. 56, 2359-2373 (2013).

\section{Figures}




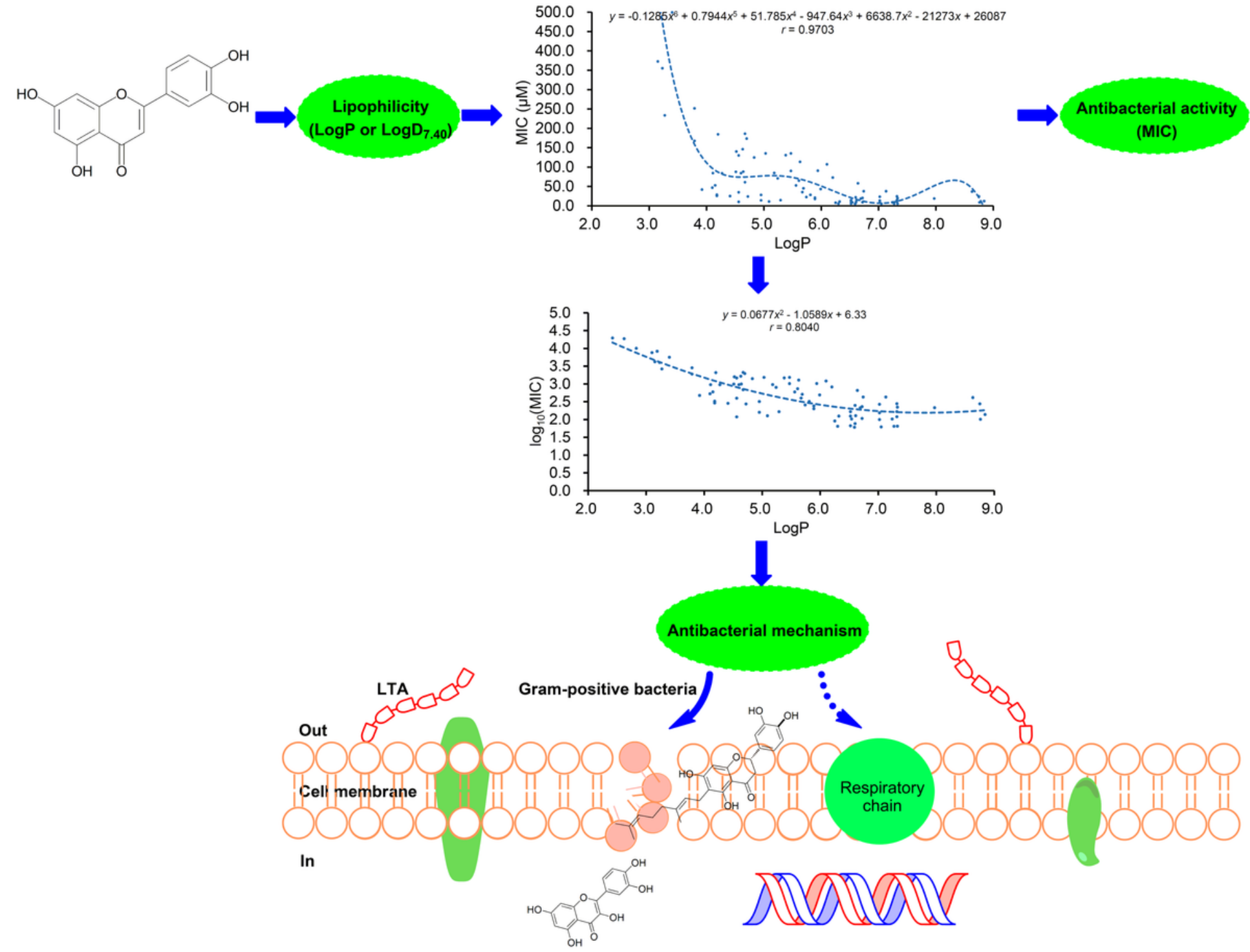

\section{Figure 1}

(Author titled this "Figure 1") Diagram for the antibacterial activities and mechanisms of plant flavonoids to gram-positive bacteria.
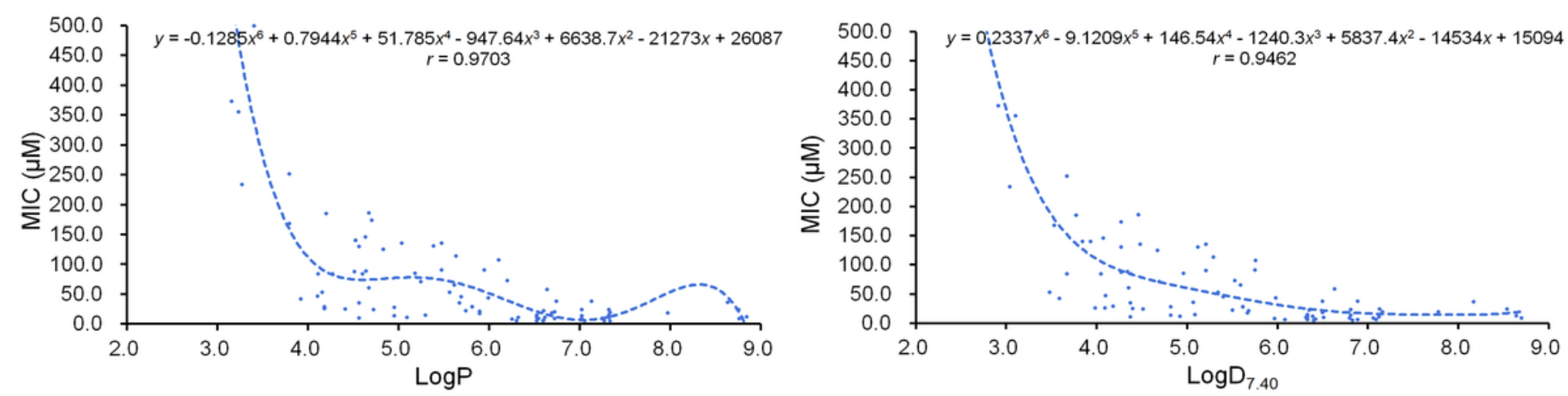

Figure 2 
(Author also titled this "Figure 1") Polynomial regression analyses for the physicochemical parameter $\operatorname{LogP}$ or $\log _{7.40}(x)$ and the MIC $(y)$ to gram-positive bacteria mainly including Staphylococcus aureus, $S$. epidermidis, or/and Bacillus subtilis, of ninety-two plant flavonoids.
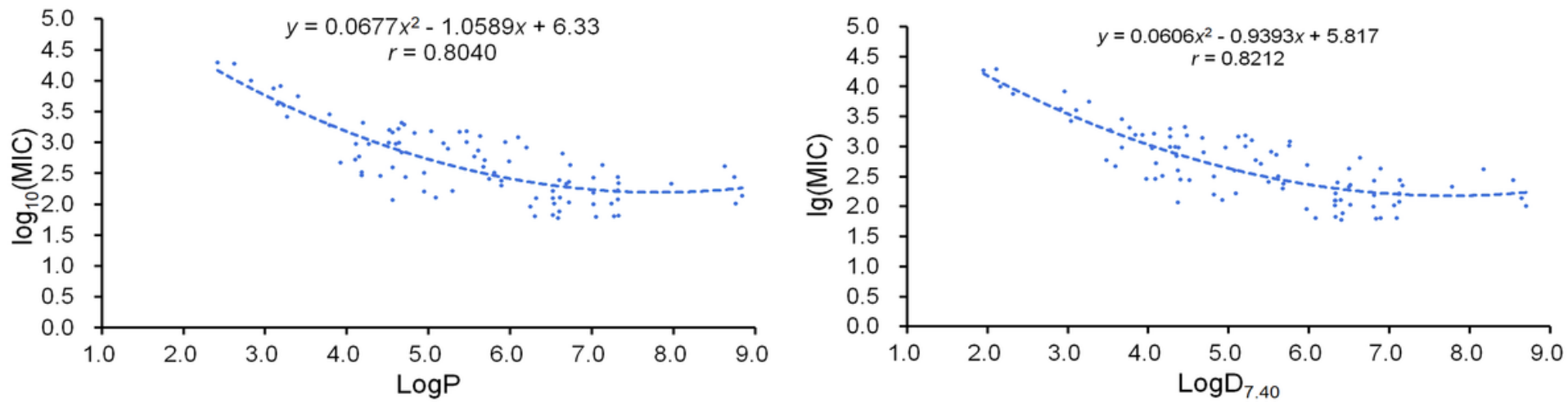

\section{Figure 3}

(Author titled this "Figure 2") Polynomial regression analyses for the physicochemical parameter LogP or $\log _{7.40}(x)$ and the $\log _{10}(\mathrm{MIC})(y)$ to gram-positive bacteria, of ninety-two plant flavonoids. 